\title{
Surgical outcome and prognostic factors influencing visual acuity in myopic foveoschisis patients
}

\author{
Dong Hyun Lee ${ }^{1}$ Inhee Moon ${ }^{1} \cdot$ Hyun Goo Kang ${ }^{1}$ - Eun Young Choi ${ }^{1} \cdot$ Sung Soo Kim² ${ }^{2}$ Suk Ho Byeon ${ }^{2}$. \\ Hyoung Jun $\mathrm{Koh}^{2} \cdot$ Sung Chul Lee $\mathbb{1}^{2} \cdot$ Min Kim$^{1}$
}

Received: 30 November 2018 / Revised: 30 March 2019 / Accepted: 23 April 2019 / Published online: 16 May 2019

(c) The Royal College of Ophthalmologists 2019

\begin{abstract}
Background To analyze the optical coherence tomography (OCT) characteristics and visual outcome of vitrectomy in myopic foveoschisis (MF) patients and identify prognostic factors.

Methods This study is a retrospective clinical cohort study in tertiary care hospital. Thirty-two eyes of 32 patients who underwent MF-related vitrectomy in were investigated retrospectively. Best-corrected visual acuity (BCVA) measured at 1 year post surgery and changes in central foveal thickness (CFT) and co-existing macular pathologies, such as foveal detachment (FD), lamellar holes, and macular holes were the main outcome measures. Prognostic factors were identified using multivariate linear regression analysis.

Results Average BCVA (in logarithm of the minimum angle of resolution) and mean CFT had improved from $0.46 \pm 0.06$ to $0.37 \pm 0.07(P=0.089)$ and from $485.72 \pm 164.69$ to $341.71 \pm 109.70(P<0.001)$, respectively. Univariate analysis identified baseline BCVA, epiretinal membrane, no coexisting OCT features other than MF, FD on OCT, and gas injection as significantly associated with visual outcome $(P<0.001,0.014,0.022,<0.001$, and 0.030$)$. Better baseline BCVA and absence of FD on OCT remained significant $(P<0.001$ and $<0.001$, respectively) after multivariate analysis.

Conclusion Good preoperative visual acuity and absence of FD pre-surgery are important predictors of good visual prognosis. Thus, timely surgical intervention, before development of macular complications, may improve visual outcome after surgical treatment of patients with MF.
\end{abstract}

\section{Introduction}

Myopic foveoschisis (MF) is a sight-threatening complication of pathological myopia or high myopia that has increased in recent years [1]. MF occurs when the neural retina separates into the outer layer and inner layers. Diagnosis of MF is confirmed by the separation between thin outer layer and thick inner layer and the presence of intraretinal cysts or intraretinal columns [2,3]. It is thought that incomplete or anomalous posterior vitreous detachment

Min Kim

minkim76@gmail.com

1 Department of Ophthalmology, Gangnam Severance Hospital, Yonsei University College of Medicine, 211, Eonjuro, Gangnamgu, Seoul, Republic of Korea

2 Department of Ophthalmology, Severance Hospital, Yonsei University College of Medicine, 50-1, Yonseiro, Seodaemun-gu, Seoul, Republic of Korea
(PVD) is related to the pathogenesis of MF, and contraction of the cortical vitreous can induce separation of the retinal layers [4]. Several studies have reported complications that may occur during the progression of MF [1,2]; a variety of complications, such as macular hole $(\mathrm{MH})$ and retinal detachment (RD) could lead to visual loss. High myopia, which can cause various ocular complications, is highly prevalent in East Asia, especially in South Korea [5], Taiwan [6], and China [7]. The prevalence of MF varies, from $9.0 \%$ to $20.0 \%$ of highly myopic populations [8, 9]. Therefore, the clinical importance of MF is not negligible.

Optical coherence tomography (OCT) is a non-invasive tool that allows detection and evaluation of various vitreoretinal diseases [10]. In particular, in highly myopic patients, the prevalence of many vitreoretinal diseases, including myopic traction maculopathy, and full-thickness $\mathrm{MH}$, is high, and OCT has an important role in the diagnosis and follow-up of such diseases.

In patients with MF, the progressive detachment of the fovea or the development and progression of $\mathrm{MH}$ can lead 
to visual decline. Thus, determining the factors predicting this outcome could assist in achieving favorable visual outcomes when performing surgery for MF. Several studies have examined important prognostic factors related to visual outcome. Preoperative visual acuity [11] and various OCT findings, as well as operative techniques, have been identified as prognostic factors in this respect. For example, preoperative ellipsoid disruption and thinner central foveal thickness (CFT) are known to be useful OCT findings [12]. However, the usefulness of gas tamponade or ILM peeling remains controversial [13-15], and the role of additional factors remain unclear.

Therefore, we here attempted to identify prognostic factors of MF by analyzing the relationship between preoperative OCT findings and best-corrected visual acuity (BCVA) in MF patients after vitrectomy, and to determine potential prognostic factors influencing visual outcome. Furthermore, we attempted to compare the effect of different surgical techniques on surgical outcome, such as intravitreal gas tamponade or ILM peeling.

\section{Methods}

This single-center study investigated 32 eyes of 32 patients who underwent pars plana vitrectomy (PPV) for MF from January 2005 to December 2016, retrospectively. All patients were of Korean ethnicity. The study was approved by the Institutional Review Board (IRB) of Yonsei University Severance Hospital (IRB approval number: 3-20180076) and was conducted in accordance with the tenets of the Declaration of Helsinki. Informed consent was obtained from all patients. The inclusion criteria were as follows: (1) patients who were diagnosed with MF; (2) patients who had received PPV for treatment of MF; (3) who had a spherical equivalent (SE) refractive error $>-6.0$ diopters (D) and/or axial length longer than $26.5 \mathrm{~mm}$ or more. The exclusion criteria were as follows: (1) history of PPV or ocular trauma; (2) presence of advanced glaucoma, amblyopia, or dense cataract.

Thorough ophthalmic examinations were performed in each patient. BCVA, based on a Snellen chart, and intraocular pressure, confirmed by non-contact tonometer, were examined. Slit-lamp examination and dilated funduscopic examination, and fundus photography (FP) were performed. All patients underwent serial spectral-domain (SD) OCT (Spectralis, Heidelberg Engineering, Heidelberg, Germany) examinations, before and 1 year after surgery. Preoperative axial length was measured using IOLMaster (Carl Zeiss Meditec, Dublin, CA, USA). All patients had undergone PPV and additional surgical procedures such as ILM peeling were also performed, depending on the patient's comorbidities. Patients were grouped according to the method of surgery received: PPV with gas injection, internal limiting membrane (ILM) peeling, or combined cataract surgery. During surgery, intravitreous injection of triamcinolone acetonide for confirmation of PVD and vitreoschisis, or indocyanine green (ICG) staining was performed for ILM peeling. Gas injection was performed using either sulfur hexafluoride $\left(\mathrm{SF}_{6}\right)$ or perfluoropropane $\left(\mathrm{C}_{3} \mathrm{~F}_{8}\right)$. The primary outcome was the final BCVA measured at 1 year post surgery. Secondary outcome included the CFT and the foveal detachment (FD) from SD-OCT in preoperative examinations at 1 year post surgery.

Paired $t$ tests and McNemar tests were used to confirm the statistical significance of changes in BCVA, CFT, and various OCT findings after PPV. Both univariate and multivariate linear regression analyses were performed to assess the factors predicting visual improvement. Subgroup analysis was used to assess the efficacy of gas tamponade and ILM peeling, using a Mann-Whitney $U$ test, Fisher's exact test, and McNemar test. Statistical analyses were performed using SPSS version 23.0 software (IBM Corp., Armonk, NY, USA). A $P$ value of $<0.05$ was considered to be statistically significant.

\section{Results}

There were 32 eyes from 32 patients who fulfilled the inclusion criteria. Six patients were male, and 26 patients were female. The mean age of patients was $62.78 \pm 2.50$ years. The mean SE refractive error was $-6.56 \pm 1.32 \mathrm{D}$. Average axial length at baseline visit was $29.32 \pm 2.31 \mathrm{~mm}$. Baseline BCVA in logarithm of the minimum angle of resolution (log MAR) was $0.37 \pm 0.07$, and CFT was $341.71 \pm 109.70 \mathrm{~mm}$. OCT examination showed that FD was present in eight patients $(25.0 \%)$. Three patients $(9.4 \%)$ had $\mathrm{MH}$, and two patients $(6.2 \%)$ had lamellar hole, as confirmed by OCT examination. Epiretinal membrane was present in 16 patients $(50.0 \%)$. In 14 patients, no other coexistent OCT findings except for MF-associated characteristic separation of outer and inner retinal layer was observed (43.8\%) (Table 1).

All patients had undergone PPV. Thirteen patients $(40.6 \%)$ had received intravitreal injection of triamcinolone acetonide, and ILM peeling using ICG dye was performed in 26 patients $(81.3 \%)$. Gas injection was performed in eight patients. Specifically, one patient $(3.1 \%)$ underwent $\mathrm{SF}_{6}$ injection, and seven patients $(21.9 \%)$ received $\mathrm{C}_{3} \mathrm{~F}_{8}$ injection. Eighteen patients $(56.3 \%)$ received cataract surgery. After PPV, various preoperative parameters had improved. More specifically, baseline BCVA, CFT, and preoperative OCT findings improved. In particular, those eyes with thicker CFT $(P<0.001)$, epiretinal membrane $(P<0.001)$, no coexisting OCT features other than MF $(P<0.001)$, or 
Table 1 Demographics and characteristics of the patients with myopic foveoschisis at baseline visit

\begin{tabular}{ll}
\hline Number of patients (eyes) & $32(32)$ \\
Age (years) & $62.78 \pm 2.50$ \\
Sex (male/female) & $6 / 26$ \\
Spherical equivalent refractive error (D) & $-6.56 \pm 1.32$ \\
Baseline axial length (mm) & $29.32 \pm 2.31$ \\
Baseline BCVA (log MAR) & $0.46 \pm 0.06$ \\
Baseline CFT ( $\mu$ m) & $485.72 \pm 164.69$ \\
Preoperative OCT findings-other features associated with MF \\
Epiretinal membrane & $16(50.0 \%)$ \\
Foveal detachment & $8(25.0 \%)$ \\
Macular hole & $3(9.4 \%)$ \\
Lamellar hole & $2(6.2 \%)$ \\
No coexisting OCT features other than MF & $14(43.8 \%)$ \\
Mean follow-up period (months) & $17.31 \pm 8.61$ \\
\hline
\end{tabular}

$D$ diopters, $B C V A$ best-corrected visual acuity, $\log M A R$ logarithm of the minimum angle of resolution, $C F T$ central foveal thickness, $O C T$ optical coherence tomography, $M F$ myopic foveoschisis. All values are shown as the mean \pm standard deviation

Table 2 Parameters before and after surgery for myopic foveoschisis

\begin{tabular}{|c|c|c|c|}
\hline Findings & Baseline & Postoperative 1 year & $P$ value \\
\hline BCVA (log MAR) & $0.46 \pm 0.06$ & $0.37 \pm 0.07$ & $0.089^{\mathrm{a}}$ \\
\hline $\mathrm{CFT}(\mu \mathrm{m})$ & $485.72 \pm 164.69$ & $341.71 \pm 109.70$ & $<0.001^{\mathrm{a}}$ \\
\hline \multicolumn{4}{|c|}{ OCT findings—other features associated with MF } \\
\hline Epiretinal membrane (\%) & $16(50.00 \%)$ & $1(3.13 \%)$ & $<0.001^{\mathrm{b}}$ \\
\hline Foveal detachment (\%) & $8(25.00 \%)$ & $1(3.13 \%)$ & $0.016^{\mathrm{b}}$ \\
\hline Macular hole $(\%)$ & $3(9.38 \%)$ & $1(3.13 \%)$ & $0.625^{\mathrm{b}}$ \\
\hline Lamellar hole (\%) & $2(6.25 \%)$ & $0(0.00 \%)$ & $0.500^{\mathrm{a}}$ \\
\hline $\begin{array}{l}\text { No coexisting OCT } \\
\text { features other } \\
\text { than MF }(\%)\end{array}$ & $14(43.75 \%)$ & $0(0.00 \%)$ & $<0.001^{\mathrm{b}}$ \\
\hline
\end{tabular}

$B C V A$ best-corrected visual acuity, $\log M A R$ logarithm of the minimum angle of resolution, $C F T$ central foveal thickness, $O C T$ optical coherence tomography. All values are shown as the mean \pm standard deviation

${ }^{\text {aP }}$ Paired $t$ test

${ }^{\mathrm{b}} \mathrm{McNemar}$ test

FD $(P=0.016)$ on OCT showed statistically significant improvement after surgery (Table 2).

To determine the effect of either gas injection or ILM peeling, subgroup analysis was performed. There was no statistically significant difference in baseline and postoperative BCVA and CFT between the gas-treated group and the no-gas-treated group (Table 3a). CFT had improved after PPV in both groups, but the degree of improvement was not statistically significant in gastreated group $(P=0.078$, vs $P<0.001$ in no-gas-treated group, Table $3 \mathrm{a})$. The proportion of FD at baseline and after surgery showed no statistically significant difference between these two groups $(P=0.378$ and 0.999 , Table 3a). Moreover, the change in BCVA, CFT, and improvement in FD showed no statistically significant difference between the gas-treated and no-gas-treated group $(P=0.104,0.815$, and 0.125 , respectively, Table 3a). A similar trend was observed between the ILM-peeled group and no-ILM-peeled group. There was no statistically significant difference in the baseline and postoperative BCVA and CFT between the ILMpeeled group and no-ILM-peeled group (Table 3b). CFT had improved significantly after PPV in the ILM-peeled group, whereas no significant improvement was noted in no-ILM-peeled group $(P<0.001 \quad$ vs $P=0.625$, Table $3 b)$. The proportion of FD at baseline and after surgery was not statistically significantly different between these two groups (All $P=0.999$, Table 3b). Moreover, the change in BCVA, CFT, and improvement in FD showed no significant differences between the ILM-peeled and no-ILM-peeled groups $(P=0.614$, 0.335 , and 0.999, respectively; Table 3b). Most of the patients had no postoperative complications, such as MH. However, a few postoperative complications were noted: central FD in one case, epiretinal membrane in one case, and rhegmatogenous $\mathrm{RD}$ in one case after 1 year.

To identity the potential prognostic factors affecting the visual outcome after surgery, linear regression analyses were performed. At first, possible prognostic factors were classified into three categories, as follows: demographic factors (age of patients), patient-related factors (SE refractive error, baseline BCVA, CFT, axial length, and presence of FD on OCT) and surgical factors (intraoperative gas injection, combined cataract surgery, and ILM peeling). After univariate linear regression analysis, baseline BCVA, presence of epiretinal membrane, FD on OCT, no coexisting OCT features other than MF, and use of gas injection were found to be statistically significant parameters associated with postoperative visual outcome $(P<$ $0.001,0.014,<0.001,0.022$, and 0.030 , respectively; Table 4). Multivariate linear regression analysis was performed to evaluate the association between these significant factors and postoperative visual acuity. Only preoperative poor BCVA and the presence of FD on OCT exam remained statistically significantly associated with poorer postoperative BCVA $(P<0.001$ and $<0.001$, respectively; Table 4).

We present a representative case of a male patient with MF. This 61-year-old male was diagnosed with MF. His SE refractive error was $-12.25 \mathrm{D}$, and his baseline $\mathrm{BCVA}$ was $1.7 \log$ MAR. His preoperative CFT was $899 \mu \mathrm{m}$. OCT examination showed a marked splitting of the fovea with posterior staphyloma (Fig. 1a). PPV with ILM peeling was 
Table 3 Comparison of parameters before and after surgery for myopic foveoschisis between A. gas-treated vs. nogas-treated group and B. ILMpeeled vs. no-ILM-peeled group

\begin{tabular}{|c|c|c|c|c|c|}
\hline \multicolumn{6}{|l|}{ A } \\
\hline Findings & $\begin{array}{l}\text { Gas-treated group } \\
(N=8)\end{array}$ & $P$ value $^{\mathrm{a}}$ & $\begin{array}{l}\text { No-gas-treated } \\
\text { group }(N=24)\end{array}$ & $P$ value $^{\mathrm{a}}$ & $P$ value $^{\mathrm{b}}$ \\
\hline Baseline BCVA (log MAR) & $0.61 \pm 0.40$ & & $0.41 \pm 0.07$ & & $0.237^{\mathrm{d}}$ \\
\hline Postoperative BCVA (log MAR) & $0.61 \pm 0.43$ & & $0.28 \pm 0.33$ & & $0.078^{\mathrm{d}}$ \\
\hline $\begin{array}{l}\text { BCVA change after } \\
\text { surgery [postoperative BCVA - } \\
\text { baseline BCVA] (log MAR) }\end{array}$ & $0.00 \pm 0.16$ & $0.563^{\mathrm{f}}$ & $-0.13 \pm 0.37$ & $0.109^{\mathrm{g}}$ & $0.104^{\mathrm{d}}$ \\
\hline Baseline CFT $(\mu \mathrm{m})$ & $508.13 \pm 224.90$ & & $478.25 \pm 148.70$ & & $0.654^{\mathrm{d}}$ \\
\hline Postoperative CFT ( $\mu \mathrm{m})$ & $316.60 \pm 104.42$ & & $349.56 \pm 116.90$ & & $0.398^{\mathrm{d}}$ \\
\hline $\begin{array}{l}\text { CFT change after } \\
\text { surgery [postoperative CFT } \\
\text { - baseline CFT] }(\mu \mathrm{m})\end{array}$ & $-193.63 \pm 259.37$ & $0.078^{\mathrm{f}}$ & $-133.58 \pm 132.90$ & $<0.001^{\mathrm{g}}$ & $0.815^{\mathrm{d}}$ \\
\hline Baseline FD (\%) & $3(37.5 \%)$ & & $5(20.8 \%)$ & & $0.378^{\mathrm{e}}$ \\
\hline Postoperative FD (\%) & $0(0.0 \%)$ & & $1(4.2 \%)$ & & $0.999^{\mathrm{e}}$ \\
\hline $\begin{array}{l}\text { Resolution of FD after } \\
\text { surgery }(\%)\end{array}$ & $100.00 \%$ & $0.125^{\mathrm{h}}$ & $80.00 \%$ & $0.125^{\mathrm{h}}$ & $0.125^{\mathrm{h}}$ \\
\hline
\end{tabular}

B

\begin{tabular}{|c|c|c|c|c|c|}
\hline Findings & $\begin{array}{l}\text { ILM-peeled } \\
\text { group } \\
(N=27)\end{array}$ & $P$ value $^{\mathrm{a}}$ & $\begin{array}{l}\text { No-ILM-peeled } \\
\text { group }(N=5)\end{array}$ & $P$ value $^{\mathrm{a}}$ & $P$ value $^{\mathrm{c}}$ \\
\hline Baseline BCVA (log MAR) & $0.41 \pm 0.31$ & & $0.74 \pm 0.43$ & & $0.087^{\mathrm{d}}$ \\
\hline $\begin{array}{l}\text { Postoperative BCVA } \\
\text { (log MAR) }\end{array}$ & $0.33 \pm 0.35$ & & $0.54 \pm 0.51$ & & $0.479^{\mathrm{d}}$ \\
\hline $\begin{array}{l}\text { BCVA change after } \\
\text { surgery [postoperative BCVA - } \\
\text { baseline BCVA] (log MAR) }\end{array}$ & $-0.07 \pm 0.31$ & $0.227^{\mathrm{f}}$ & $-0.20 \pm 0.45$ & $0.999^{\mathrm{g}}$ & $0.614^{\mathrm{d}}$ \\
\hline Baseline CFT $(\mu \mathrm{m})$ & $481.52 \pm 152.19$ & & $508.40 \pm 256.28$ & & $0.479^{\mathrm{d}}$ \\
\hline Postoperative CFT $(\mu \mathrm{m})$ & $324.72 \pm 106.78$ & & $443.67 \pm 106.11$ & & $0.101^{\mathrm{d}}$ \\
\hline $\begin{array}{l}\text { CFT change after } \\
\text { surgery [postoperative CFT - } \\
\text { baseline CFT] }(\mu \mathrm{m})\end{array}$ & $\begin{array}{l}-151.78 \pm \\
143.27\end{array}$ & $<0.001^{\mathrm{f}}$ & $-131.40 \pm 301.41$ & $0.625^{\mathrm{g}}$ & $0.335^{\mathrm{d}}$ \\
\hline Baseline FD (\%) & $7(25.9 \%)$ & & $1(20.0 \%)$ & & $0.999^{\mathrm{e}}$ \\
\hline Postoperative FD (\%) & $1(3.7 \%)$ & & $0(0.0 \%)$ & & $0.999^{\mathrm{e}}$ \\
\hline $\begin{array}{l}\text { Resolution of FD after } \\
\text { surgery }(\%)\end{array}$ & $85.70 \%$ & $0.031^{\mathrm{h}}$ & $100.00 \%$ & $0.999^{\mathrm{h}}$ & $0.999^{\mathrm{h}}$ \\
\hline
\end{tabular}

$\overline{B C V A}$ best-corrected visual acuity, $\log M A R$ logarithm of the minimum angle of resolution, $C F T$ central foveal thickness, $F D$ foveal detachment

${ }^{\mathrm{a}}$ Comparison between baseline and postoperative values

${ }^{\mathrm{b}}$ Comparison between gas-treated group and no-gas-treated group

${ }^{\mathrm{c}}$ Comparison between ILM-peeled group and no-ILM-peeled group

${ }^{\mathrm{d}}$ Mann-Whitney $U$ test

'Fisher's exact test

${ }^{\mathrm{f}}$ Wilcoxon signed-rank test

${ }^{\mathrm{g}}$ Paired $t$ test

${ }^{\mathrm{h}} \mathrm{McNemar}$ test 
Table 4 Correlations between preoperative factors and visual acuity at 12 months after surgery, based on univariate and multivariate linear regression analysis

\begin{tabular}{|c|c|c|c|c|}
\hline & \multicolumn{2}{|c|}{ Univariate linear regression analysis } & \multicolumn{2}{|c|}{ Multivariate linear regression analysis } \\
\hline & Coefficient & $P$ value & Coefficient & $P$ value \\
\hline \multicolumn{5}{|l|}{ Demographic factors } \\
\hline Age & 0.007 & 0.152 & & \\
\hline \multicolumn{5}{|l|}{ Patient-related parameters } \\
\hline Spherical equivalent refractive error (D) & 0.007 & 0.455 & & \\
\hline Baseline BCVA (log MAR) & 0.634 & $<0.001$ & 0.505 & $<0.001$ \\
\hline Baseline CFT & 0.001 & 0.063 & & \\
\hline Baseline axial length & 0.05 & 0.088 & & \\
\hline \multicolumn{5}{|c|}{ Preoperative OCT findings - other features associated with MF } \\
\hline Epiretinal membrane & -0.319 & 0.014 & -0.031 & 0.814 \\
\hline Foveal detachment & 0.563 & $<0.001$ & 0.476 & $<0.001$ \\
\hline Macular hole & -0.072 & 0.757 & & \\
\hline Lamellar hole & 0.037 & 0.896 & & \\
\hline No coexisting OCT features other than MF & 0.302 & 0.022 & 0.032 & 0.804 \\
\hline \multicolumn{5}{|l|}{ Surgical factors } \\
\hline Gas injection & 0.329 & 0.030 & 0.186 & 0.109 \\
\hline Combined cataract surgery & -0.023 & 0.867 & & \\
\hline ILM peeling & -0.288 & 0.091 & & \\
\hline
\end{tabular}

$D$ Diopters, $B C V A$ best-corrected visual acuity, $\log M A R$ logarithm of the minimum angle of resolution, $C F T$ central foveal thickness, $O C T$ optical coherence tomography, $M F$ myopic foveoschisis, ILM internal limiting membrane

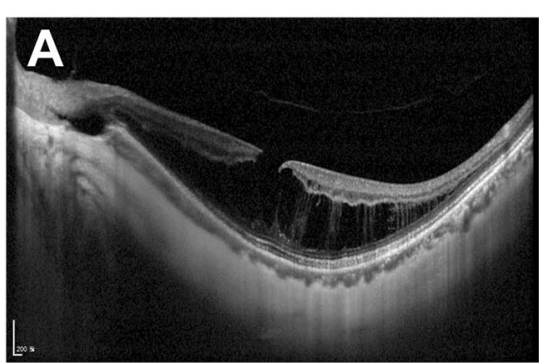

Fig. 1 This 61-year-old male had MF with FD. His spherical equivalent refractive error was $-12.25 \mathrm{D}$, and his baseline BCVA was $1.7 \log$ MAR. a The preoperative OCT examination showed a marked splitting of the fovea with posterior staphyloma. b He had received PPV with ILM peeling and $\mathrm{SF}_{6}$ gas tamponade. After 1 year, OCT examination revealed resolution of foveoschisis. His BCVA improved

performed. Gas tamponade was performed with $\mathrm{SF}_{6}$. After 1 year, OCT examination revealed resolution of foveoschisis (Fig. 1b). His BCVA had improved from $1.7 \log$ MAR to $1.0 \log$ MAR.

\section{Discussion}

The results of this study show that patients with MF had various comorbidities influencing vision, and that PPV itself

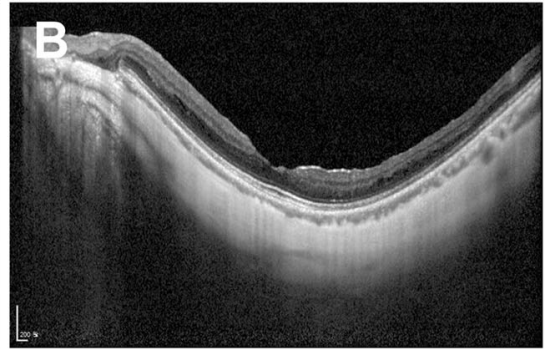

to $1.0 \log$ MAR. MF: myopic foveoschisis. FD: foveal detachment. D: diopters. BCVA: best-corrected visual acuity. log MAR: logarithm of the minimum angle of resolution. OCT: optical coherence tomography. PPV: pars plana vitrectomy. ILM: internal limiting membrane. $\mathrm{SF}_{6}$ : sulfur hexafluoride

could effectively treat MF and its associated FD status. Among various preoperative conditions and surgical interventions, preoperative low BCVA and the presence of FD were shown to be associated with poor visual outcome.

Vitrectomy with peeling of ILM and gas tamponade is the current mainstay for treatment of MF [14]. Recently, several reports have suggested various treatment option for treatment of MF. Zhu et al. [16] showed that gas tamponade using $\mathrm{C}_{3} \mathrm{~F}_{8}$ and laser photocoagulation without PPV could improve the thickness of the macula and BCVA $(P=0.001$ 
and 0.87 , respectively). In addition, a recent study has reported that the macular buckle technique produces better results than PPV and results in fewer incidences of complications such as RD or secondary MH [17]. In addition, the therapeutic effect of posterior scleral reinforcement in treating MF has been reported [18]. Yun et al. [13] reported that, even though vitrectomy without gas tamponade is as effective as vitrectomy with gas tamponade, eyes treated with gas tamponade achieved more rapid resolution of MF. Some studies have shown a favorable outcome even without gas tamponade [15, 19], but intraoperative gas tamponade proved to be useful, because it has been shown that MF recovery is faster when gas tamponade is used [20]. Our study showed that PPV with gas tamponade may be related to better visual outcome, confirmed by univariate linear regression analysis. However, the effect of ILM peeling on postoperative visual prognosis was not statistically significant. These results suggest that removal of vitreomacular traction is perhaps more important than ILM peeling for the treatment of MF. The usefulness of ILM peeling has been discussed in several articles. Lim et al. [19] reported that vitrectomy with ILM peeling without gas tamponade can produce good results. However, Qi et al. [21] showed that vitrectomy with gas tamponade alone can obtain good results without ILM peeling. Moreover, during peeling of ILM, the fovea may suffer surgical trauma in highly myopic patients, which may lead to decreased postoperative visual acuity. In fact, it has been shown that fovea-sparing ILM peeling can prevent the occurrence of postoperative MH [22], Therefore, it is conceivable that ILM peeling may not have had a significant impact on postoperative BCVA.

In the present study, good baseline BCVA and absence of FD on preoperative OCT examination were found to predict good postoperative visual outcome. Intraoperative gas tamponade itself could contribute to a better prognosis, but compared with other factors, the effect on visual outcome was relatively small. Several articles estimating prognostic factors have recently been published [11, 12, 23]. Lim et al. [12] demonstrated that preoperative ellipsoid disruption and thinner CFT were related to poor prognosis after PPV in MF patients. Lehmann et al. [11] reported that preoperative visual acuity is the main factor influencing final visual outcome, whereas Fujimoto et al. [23] suggested that retinal thickness changes and recovery of photoreceptor cells are important OCT findings related to final BCVA. We analyzed several possible prognostic factors that were mentioned in other previous studies. Moreover, those possible prognostic factors were analyzed collectively to find the most significant factors influencing the postoperative visual outcome. In this study, good baseline BCVA and absence of FD were the most important, but a large population-based study is needed because of relatively small study populations in this study. In addition, because of the difficulty in performing PPV surgery in patients with high myopia and posterior staphyloma, the effect of the operator's surgical skills on the outcome is not negligible and should also be taken into consideration.

Some complications occurred in our patients during the follow-up period. One 68-year-old female patient whose axial length was $31.41 \mathrm{~mm}$ had received $\mathrm{PPV}$ with $\mathrm{C}_{3} \mathrm{~F}_{8}$ gas injection for treatment of full-thickness $\mathrm{MH}$ initially. She developed rhegmatogenous RD, which was treated with PPV for residual peripheral vitreous, endolaser photocoagulation, and silicone oil tamponade. MF recurred in one patient whose CFT was $802 \mu \mathrm{m}$ at preoperative examination. This patient had been diagnosed with epiretinal membrane, but he did not undergo ILM peeling during PPV. Many studies have reported the usefulness of ILM peeling $[15,24]$. It has also been reported that fovea-sparing ILM peeling can be an effective approach for treating MF without risk of MH [24]. Huang et al. [25] found that the prevalence of secondary MH RD after PPV without ILM peeling is high in patients with preoperative FD. To prevent possible complications, including epiretinal membrane and $\mathrm{MH}$, fovea-sparing ILM peeling may be considered as an option for the treatment of MF.

Our study had some limitations. First, it was a retrospective study. In addition, study population is not large. The patients with MF in this study had various accompanying comorbidities. As this population was predominantly elderly, further studies are needed to determine whether these findings also apply to non-elderly patients.

In conclusion, in the treatment of patients with MF, removal of vitreomacular traction by PPV surgery may be helpful in relieving MF and resolving FD. We show that, among various preoperative and surgical factors, good visual acuity before surgery and absence of FD are important for good visual outcomes, and that preoperative low BCVA and the presence of FD are associated with poor visual outcome. Thus, timely surgical intervention may be helpful in achieving successful visual outcomes in patients with MF.

\section{Summary}

\section{What was known before}

- Several factors have been studied to determine the visual prognosis of MF.

- Preoperative visual acuity, various OCT findings such as ellipsoid disruption and thinner CFT, and surgical methods like gas tamponade and ILM peeling were found to be important for prognosis, but some factors were debated. 


\section{What this study adds}

- Our study shows that good baseline BCVA and absence of FD on preoperative OCT examination are important for visual prognosis.

- Thus, timely surgical intervention may be helpful in achieving successful visual outcomes in patients with MF.

Acknowledgements This study was supported by Yonsei University Faculty Research Grant (6-2017-0149).

\section{Compliance with ethical standards}

Conflict of interest The authors declare that they have no conflict of interest.

Publisher's note: Springer Nature remains neutral with regard to jurisdictional claims in published maps and institutional affiliations.

\section{References}

1. Shimada N, Ohno-Matsui K, Baba T, Futagami S, Tokoro T, Mochizuki M. Natural course of macular retinoschisis in highly myopic eyes without macular hole or retinal detachment. Am J Ophthalmol. 2006;142:497-500.

2. Benhamou N, Massin P, Haouchine B, Erginay A, Gaudric A. Macular retinoschisis in highly myopic eyes. Am J Ophthalmol. 2002;133:794-800.

3. Sayanagi K, Morimoto Y, Ikuno Y, Tano Y. Spectral-domain optical coherence tomographic findings in myopic foveoschisis. Retina. 2010;30:623-8.

4. Gohil R, Sivaprasad S, Han LT, Mathew R, Kiousis G, Yang Y. Myopic foveoschisis: a clinical review. Eye (Lond). 2015;29:593-601.

5. Rim TH, Kim SH, Lim KH, Choi M, Kim HY, Baek SH Refractive errors in Koreans: the Korea National Health and Nutrition Examination Survey 2008-2012. Korean J Ophthalmol. 2016;30:214-24.

6. Lin LL, Shih YF, Hsiao CK, Chen CJ. Prevalence of myopia in Taiwanese schoolchildren: 1983 to 2000. Ann Acad Med Singapore. 2004;33:27-33.

7. Guo K, Yang DY, Wang Y, Yang XR, Jing XX, Guo YY, et al. Prevalence of myopia in schoolchildren in Ejina: the Gobi Desert Children Eye Study. Invest Ophthalmol Vis Sci. 2015;56:1769-74.

8. Baba T, Ohno-Matsui K, Futagami S, Yoshida T, Yasuzumi K, Kojima A, et al. Prevalence and characteristics of foveal retinal detachment without macular hole in high myopia. Am J Ophthalmol. 2003;135:338-42.

9. Panozzo G, Mercanti A. Optical coherence tomography findings in myopic traction maculopathy. Arch Ophthalmol. 2004;122:1455-60.
10. Lee DH, Kang HG, Lee SC, Kim M. Features of optical coherence tomography predictive of choroidal neovascularisation treatment response in pathological myopia in association with fluorescein angiography. $\mathrm{Br} \quad \mathrm{J}$ Ophthalmol. 2018;102:238-42.

11. Lehmann M, Devin F, Rothschild PR, Gaucher D, Morin B, Philippakis E, et al. Preoperative factors influencing visual recovery after vitrectomy for myopic foveoschisis. Retina. 2017;39:594-600.

12. Lim LS, Ng WY, Wong D, Wong E, Yeo I, Ang CL, et al. Prognostic factor analysis of vitrectomy for myopic foveoschisis. Br J Ophthalmol. 2015;99:1639-43.

13. Yun LN, Xing YQ. Long-term outcome of highly myopic foveoschisis treated by vitrectomy with or without gas tamponade. Int J Ophthalmol. 2017;10:1392-5.

14. Meng B, Zhao L, Yin Y, Li H, Wang X, Yang X, et al. Internal limiting membrane peeling and gas tamponade for myopic foveoschisis: a systematic review and meta-analysis. BMC Ophthalmol. 2017;17:166

15. Uchida A, Shinoda H, Koto T, Mochimaru H, Nagai N, Tsubota K, et al. Vitrectomy for myopic foveoschisis with internal limiting membrane peeling and no gas tamponade. Retina. 2014;34:455-60.

16. Zhu X, Shen P, Li C, Li H, Huang H, Shi K, et al. Intravitreal gas injection with laser photocoagulation for highly myopic foveoschisis: Technique and outcome. Retina. 2018; https://doi.org/10. 1097/iae.0000000000002145.

17. Alkabes M, Mateo C. Macular buckle technique in myopic traction maculopathy: a 16-year review of the literature and a comparison with vitreous surgery. Graefes Arch Clin Exp Ophthalmol. 2018;256:863-77.

18. Li XJ, Yang XP, Li QM, Wang YY, Wang J, Lyu XB, et al. Posterior scleral reinforcement combined with vitrectomy for myopic foveoschisis. Int J Ophthalmol. 2016;9:258-61.

19. Lim SJ, Kwon YH, Kim SH, You YS, Kwon OW. Vitrectomy and internal limiting membrane peeling without gas tamponade for myopic foveoschisis. Graefes Arch Clin Exp Ophthalmol. 2012;250:1573-7.

20. Kim KS, Lee SB, Lee WK. Vitrectomy and internal limiting membrane peeling with and without gas tamponade for myopic foveoschisis. Am J Ophthalmol. 2012;153:320-6.

21. Qi Y, Duan AL, Meng X, Wang N. Vitrectomy without inner limiting membrane peeling for macular retinoschisis in highly myopic eyes. Retina. 2016;36:953-6.

22. Shimada N, Sugamoto Y, Ogawa M, Takase H, Ohno-Matsui K. Fovea-sparing internal limiting membrane peeling for myopic traction maculopathy. Am J Ophthalmol. 2012;154:693-701.

23. Fujimoto S, Ikuno Y, Nishida K. Postoperative optical coherence tomographic appearance and relation to visual acuity after vitrectomy for myopic foveoschisis. Am J Ophthalmol. 2013;156:968-73.

24. Seppey C, Wolfensberger TJ. Vitrectomy with fovea-sparing internal limiting membrane peeling for myopic foveoschisis. Klin Monbl Augenheilkd. 2017;234:497-500.

25. Huang Y, Huang W, Ng DSC, Duan A. Risk factors for development of macular hole retinal detachment after pars plana vitrectomy for pathologic myopic foveoschisis. Retina. 2017;37:1049-54. 